\title{
Study of hybrid orthonormal functions method for solving second kind fuzzy Fredholm integral equations
}

\author{
Praveen Agarwal' ${ }^{1}$ Mohamed Ramadan², Heba S. Osheba² and Yu-Ming Chu ${ }^{3,4^{*}}$
}

\section{"Correspondence:}

chuyuming2005@126.com

${ }^{3}$ Department of Mathematics,

Huzhou University, Huzhou 313000,

P.R. China

${ }^{4}$ Hunan Provincial Key Laboratory of

Mathematical Modeling and

Analysis in Engineering, Changsha

University of Science \& Technology,

Changsha 410114, China

Full list of author information is

available at the end of the article

\begin{abstract}
The approximate numerical solution of the linear second kind of fuzzy integral Fredholm equations is discussed in this article. A new approach uses hybrid functions, and some useful properties of these functions are proposed to transform linear second type fuzzy integral Fredholm equations into an algebraic equation. The new approach is a mixture of Bernstein polynomials (BPs) and enhanced block-pulse functions (IBPFs) at interval $[0,1)$. The approach is appealing and very easy to implement computationally. Some numerical tests show the reliability and exactness of the suggested scheme.
\end{abstract}

MSC: $65 T 60 ; 65 R 20 ; 45 A 05 ; 41 \mathrm{~A} 30$

Keywords: Fuzzy Fredholm integral equation; Bernstein polynomial; Improved block-pulse function; Absolute error; Accuracy

\section{Introduction}

There are many fuzzy mathematical models for the analysis of fuzzy systems with applications. The main objective of these models is to focus on how to use the model of a fuzzy system to solve fuzzy mathematics problems. Some mathematical models are based on fuzzy set theory; see [1]. In various cases of data collection one may identify situations, where measurements in a data sample are only partially associated with their underlying population. The presence of such data imposes challenges to any statistical procedure of the comparison of distributions or numerical characteristics of variables. Some work presents procedures to test the identity of distributions on the basis of one key, the socalled fuzzy samples; see for example [2]. Moreover, fuzzy systems and neural network techniques seem very well suited for typical technical problems; for example, see [3, 4] where the generalized net model is addressed to the appraisal of lecturers with intuitionistic fuzzy estimations that represent a model of a digital university.

Integral fuzzy forms equations in applied mathematics are necessary to research and solve a large proportion of the problems in different topics. Fuzzy integral equations (FIEs) topics have been of increasing interest for some time, particularly in physics, geography, medicine, biology, and fuzzy control, and have grown rapidly in recent years. Some of

(c) The Author(s) 2020. This article is licensed under a Creative Commons Attribution 4.0 International License, which permits use, sharing, adaptation, distribution and reproduction in any medium or format, as long as you give appropriate credit to the original author(s) and the source, provide a link to the Creative Commons licence, and indicate if changes were made. The images or other third party material in this article are included in the article's Creative Commons licence, unless indicated otherwise in a credit line to the material. If material is not included in the article's Creative Commons licence and your intended use is not permitted by statutory regulation or exceeds the permitted use, you will need to obtain permission directly from the copyright holder. To view a copy of this licence, visit http://creativecommons.org/licenses/by/4.0/. 
the parameters for our problems are usually set for several applications that are typically defined by fuzzy numbers rather than smooth, and therefore it is important to establish mathematical models and computational methods for appropriate care and resolution of general fuzzy integral equations. Those were studied in particular by Nanda [5], Kaleva [6], Ralescu and Adams [7], Goetschel and Voxman [8], Wang [9], and Bede and Gal [10]. Molabahrami et al. [11] recently used the parametric shape of a fuzzy number and in the crisp case they transformed a linear fuzzy integral Fredholm equation to two linear systems of second type integral equations. These equations are usually analytically difficult to solve. Therefore approximate solutions need to be found. A variety of computational methods for solving linear fuzzy Fredholm integral equations have recently been investigated. Block-pulse functions [12-17], hair functions [18], triangular functions [19-21], Chebyshev hybrids with functions of block-pulse form [22, 23], Legendre hybrid with functions of block-pulse form [24-26], hybrid Taylor, block-pulse functions [27], Fourier hybrid with functions of block-pulse form [28] and Bernstein polynomials hybrid with functions of block-pulse form [29-31]. Harmuth first introduced block-pulse functions to electrical engineering, and other scholars addressed the block pulse [32-35]. Bernstein polynomials play a prominent role in various mathematical fields. These polynomials were also used to solve integral equations, differentials and in approximation theory [36-40]. The improved block-pulse function is introduced by Farshid Mirzaee [41]. These modified block-pulse functions are applied to numerical solution of stochastic Volterra integral equations [42]. The Bernstein polynomials (BPs) and improved block-pulse functions (IBPFs) are introduced in [43].

The purpose of this article is the application of the HBIBP method, for the first time, to get an approximate solution for the second kind of linear Fredholm fuzzy equations. Finally, we test the system on a few examples to demonstrate its accuracy and effectiveness.

The paper is organized as follows: Several notes and theorems on fuzzy set structures and analysis of integral equations of fuzzy Fredholm type, improved block-pulse function and polynomials of Bernstein and its properties are briefly discussed in Sect. 2. In Sect. 3, we are launching a new blend of the Bernstein and enhanced block-pulse functions. The linear integral Fredholm equation is solved using a new basis in Sect. 4. The error estimate is given in Sect. 5 for the proposed method. Test problems to demonstrate the reliability and accuracy of the proposed scheme are considered in Sect. 6. Finally, we make our closing remarks.

\section{Preliminaries}

\subsection{Bernstein-type polynomials}

Definition ([44]) The $M$ th degree Bernstein polynomials are defined on the interval $[0,1]$ by

$$
B_{i, M}(x)=\left(\begin{array}{c}
M \\
i
\end{array}\right) x^{i}(1-x)^{M-i}, \quad i=0,1, \ldots, M,
$$

where $\left(\begin{array}{c}M \\ i\end{array}\right)=\frac{M !}{i !(M-i) !}$.

By binomial expansion of $(1-x)^{M-i}$, we get

$$
\left(\begin{array}{c}
M \\
i
\end{array}\right) x^{i}(1-x)^{M-i}=\sum_{k=0}^{M-i}(-1)^{k}\left(\begin{array}{c}
M \\
i
\end{array}\right)\left(\begin{array}{c}
M-i \\
k
\end{array}\right) x^{i+k},
$$


and we normally set for mathematical convenience $B_{i, M}=0$ if $i<0$ or $i>M$. One can also use a recursive description to generate the Bernstein polynomials over $[0,1]$ so that the $i$ th Bernstein polynomial of $M$ th degree takes the form

$$
B_{i, M}(x)=(1-x) B_{i, M-1}(x)+x B_{i-1, M-1}(x) .
$$

The Bernstein polynomials can be proved to be positive and the sum of all the Bernstein polynomials is unity, for all real $x \in[0,1]$, i.e., $\sum_{i=0}^{M} B_{i, M}(x)=1$ (unity partition property). We can easily prove that every $M$ th degree polynomial can be extended in terms of these basis functions.

\subsection{Improved block-pulse function}

Definition An $(n+1)$-set of IBPFs consists of $(n+1)$ functions that are specified over a region $D$, with a slight change in the definition of function, as follows [41]:

$$
\begin{aligned}
& \varphi_{1}(x)= \begin{cases}1, & x \in\left[0, \frac{h}{2}\right), \\
0, & \text { otherwise }\end{cases} \\
& \varphi_{i}(x)= \begin{cases}1, & x \in\left[(i-2) h+\frac{h}{2},(i-1) h+\frac{h}{2}\right), \quad i=2,3, \ldots, n, \\
0, & \text { otherwise }\end{cases} \\
& \varphi_{n+1}(x)= \begin{cases}1, & x \in\left[1-\frac{h}{2}, 1\right), \\
0, & \text { otherwise. }\end{cases}
\end{aligned}
$$

Here $n$ is an arbitrary positive integer, and $h=\frac{1}{n}$.

The most important properties for these functions are disjointness, orthogonality, and completeness.

Lemma Let a set of improved block-pulse functions (IBPFs) $\varphi_{i}(x), i=1,2, \ldots, N+1$ be defined on the interval $[0,1)$ such that

$$
\begin{aligned}
& \varphi_{1}(x)= \begin{cases}1, & x \in\left[0, \frac{h}{2}\right), \\
0, & \text { otherwise, }\end{cases} \\
& \varphi_{i}(x)= \begin{cases}1, & x \in\left[(i-2) h+\frac{h}{2},(i-1) h+\frac{h}{2}\right), \quad i=2,3, \ldots, n, \\
0, & \text { otherwise, }\end{cases} \\
& \varphi_{n+1}(x)= \begin{cases}1, & x \in\left[1-\frac{h}{2}, 1\right), \\
0, & \text { otherwise. }\end{cases}
\end{aligned}
$$

The properties of such functions correspond to the following:

(i) disconnectedness,

(ii) orthogonality,

(iii) completeness. 
Proof Using the definition of an improved block pulse, the disjointness property can be obtained as follows:

$$
\varphi_{i}(x) \varphi_{j}(x)= \begin{cases}\varphi_{i}(x), & i=j, \\ 0, & \text { otherwise }\end{cases}
$$

The other property of IBPFs is mutual orthogonality, where $x \in D$,

$$
\int_{0}^{1} \varphi_{i}(x) \varphi_{j}(x) d x= \begin{cases}\frac{h}{2}, & i=j \in\{1, n+1\} \\ h, & i=j \in\{2,3, \ldots, n\} \\ 0, & \text { otherwise. }\end{cases}
$$

For the completeness property: every $f \in L^{2}[0,1]$. If $n$ goes to infinity, Parseval's identity holds:

$$
\int_{0}^{1} f^{2}(x) d x=\sum_{i=0}^{\infty} f_{i}^{2}\left\|\phi_{i}(x)\right\|^{2}, \quad \text { where } f_{i}=\frac{1}{h} \int_{0}^{1} f(x) \phi_{i}(x) d x .
$$

\subsubsection{Vector forms of IBPFS}

Consider the first $(n+1)$ terms of IBPFs and write them concisely as $(n+1)$-vector

$$
\Phi_{n}(x)=\left[\varphi_{1}(x), \varphi_{2}(x), \ldots, \varphi_{n+1}(x)\right]^{T}, \quad x \in D,
$$

from disjointness

$$
\Phi_{n}(x) \Phi_{n}^{T}(x)=\left(\begin{array}{cccc}
\varphi_{1}(x) & 0 & \ldots & 0 \\
0 & \varphi_{2}(x) & \ldots & 0 \\
\vdots & \vdots & \ddots & \vdots \\
0 & 0 & \ldots & \varphi_{n+1}(x)
\end{array}\right)=\operatorname{diag}\left(\Phi_{n}(x)\right)
$$

\subsubsection{IBPFs expansions}

A continuous function $f(x) \in L^{2}(D)$ may be expanded by the IBPFs as

$$
f(x) \simeq f_{n}(x)=\sum_{i=1}^{n+1} f_{i} \varphi_{i}(x)=F_{n}^{T} \Phi_{n}(x)=\Phi_{n}^{T}(x) F_{n},
$$

where $F_{n}$ is a $(n+1) \times 1$ vector given by

$$
F_{n}=\left[f_{1}, f_{2}, \ldots, f_{n+1}\right]^{T},
$$

and $\Phi_{n}(x)$ is defined in (2.1) and $f_{i}$ is obtained as

$$
f_{i}= \begin{cases}2 n \int_{0}^{\frac{h}{2}} f(x) d x, & i=1, \\ n \int_{(i-2) h+\frac{h}{2}}^{(i-1) h} f(x) d x, & i=2, \ldots, n, \\ 2 n \int_{1-\frac{h}{2}}^{1} f(x) d x, & i=n+1 .\end{cases}
$$


Similarly a function of two variables $K(x, y) \in L^{2}(D \times D)$ can be approximated by IBPFs as follows:

$$
K(x, y) \simeq \Phi_{n+1}^{T}(x) K_{n} \Phi_{n+1}(y) .
$$

Here $\Phi_{n}(x)$ and $\Phi_{n}(y)$ are IBPFs vector of dimension $(n+1)$ and $K_{n}=\left[k_{i, j}\right]$ is the $(n+1) \times$ $(n+1)$ IBPFs coefficient matrix of $k(x, y)$.

\subsection{Fuzzy functions}

Now recall the following definitions needed throughout the paper.

Definition ([45]) A fuzzy number is a fuzzy set $u: R^{1} \rightarrow[0,1]$ that satisfies following condition:

- $u$ is upper semi-continuous.

- $u(x)=0$ outside the interval $[c, d]$.

- Here the real numbers $a$ and $b: c \leq a \leq b \leq d$ for which

- $u(x)$ shows rising monotony on $[c, a]$,

$-u(x)$ is monotonously declining on $[b, d]$,

$-u(x)=1, a \leq x \leq b$.

Definition ([45]) A fuzzy number $u$ is a pair $(\underline{u}(r), \bar{u}(r))$ of functions $\underline{u}(r)$ and $\bar{u}(r), 0 \leq$ $r \leq 1$, that satisfy the following requirements:

- $\underline{u}(r)$ is a bounded monotonic, continuous increasing left function,

- $\bar{u}(r)$ is a bounded monotonous decreasing continuous left function,

- $\underline{u}(r) \leq \bar{u}(r), 0 \leq r \leq 1$.

For arbitrary $u=(\underline{u}(r), \bar{u}(r)), v=(\underline{v}(r), \bar{v}(r))$ and $k>0$ we describe adding $(u+v)$ and multiplication by $k$ as follows:

- $u=v$ if and only if $\underline{u}(r)=\underline{v}(r)$ and $\bar{u}(r)+\bar{v}(r)$,

- $u \oplus v=(\underline{u}(r)+\underline{v}(r), \bar{u}(r)+\bar{v}(r))$,

$$
(\lambda \otimes u)= \begin{cases}(\lambda \underline{u}(r), \lambda \bar{u}(r)) & \lambda \geq 0, \\ (\lambda \bar{u}(r), \lambda \underline{u}(r)) & \lambda<0 .\end{cases}
$$

\subsection{Fredholm integral equations of fuzzy form}

The Fredholm fuzzy second form integral equation is

$$
\tilde{u}(x)=\tilde{f}(x)+\lambda \int_{a}^{b} k(x, t) \tilde{u}(t) d t
$$

where $>0, k(x, t)$ is an arbitrary kernel function and $\tilde{f}(x)$ and $\tilde{u}(x)$ are fuzzy functions over $[a, b]$.

The solution of Eq. (2.2) is crisp where $\tilde{f}(x)$ is a crisp function. However, if $\tilde{f}(x)$ is a fuzzy function, then Eq. (2.2) can possess only fuzzy solutions. For the implementation of the numerical method for solving Eq. (2.2), we rewrite it as

$$
\underline{u}(x, r)=f(x, r)+\lambda \int_{a}^{b} \underline{u}(t, r) d t,
$$




$$
\bar{u}(x, r)=\bar{f}(x, r)+\lambda \int_{a}^{b} \bar{U}(t, r) d t
$$

where

$$
\begin{gathered}
\underline{U}(t, r)= \begin{cases}k(x, t) \underline{u}(t, r), & k(x, t) \geq 0, \\
k(x, t) \bar{u}(t, r), & k(x, t)<0,\end{cases} \\
\bar{U}(t, r)= \begin{cases}k(x, t) \bar{u}(t, r), & k(x, t) \geq 0, \\
k(x, t) \underline{u}(t, r), & k(x, t)<0 .\end{cases}
\end{gathered}
$$

Theorem 1 Let $k(x, t)$ be continuous for $a \leq x, t \leq b$ and $\tilde{f}(x)$ be a fuzzy continuous of $x$, $a \leq x \leq b$. If $\lambda<\frac{1}{M(b-a)}$, where $M=\max _{a \leq x, t \leq b}|k(x, t)|$, then the iterative procedure

$$
\begin{aligned}
& \tilde{u}_{0}(x)=\tilde{f}(x), \\
& \vdots \\
& \tilde{u}_{k}(x)=\tilde{f}(x)+\lambda \int_{a}^{b} k(x, t) \tilde{u}_{k-1}(t) d t, \quad k \geq 1,
\end{aligned}
$$

converges to the unique solution of (2.2). Specially,

$$
\sup _{a \leq x \leq b} D\left(\tilde{u}(x), \tilde{u}_{k}(x)\right) \leq \frac{L^{k}}{1-L} \sup _{a \leq x \leq b} D\left(\tilde{u}_{0}(x), \tilde{u}_{1}(x)\right)
$$

where $L=\lambda M(b-a)$.

\section{New hybrid Bernstein improved block-pulse functions (HBIBPFs) method}

Definition ([43]) $\operatorname{HBIBP}_{i, j}(x)$ is the combination of Bernstein polynomials and improved block-pulse functions where both are complete and orthogonal, then the set is a complete orthogonal complete system. Hybrid orthonormal Bernstein and improved block-pulse functions where $j=0,1, \ldots, M, i=1,2, \ldots, N+1, \operatorname{HBIBP}_{i, j}(x)$ have two arguments; $i$ and $j$ are the order of IBPFs and degree of BPs, respectively. $\operatorname{HBIBP}(x)$ is defined on the interval $[0,1)$ as follows:

$$
\begin{aligned}
& \operatorname{HBIBP}_{i, j}(x)=\left\{\begin{array}{ll}
B_{j, M}\left(\frac{2 x}{h}\right), & x \in\left[0, \frac{h}{2}\right), \\
0, & \text { otherwise, }
\end{array} \quad \text { for } i=1, j=0,1, \ldots, M,\right. \\
& \operatorname{HBIBP}_{i, j}(x)= \begin{cases}B_{j, M}\left(\frac{x}{h}+\frac{3}{2}-i\right), & x \in\left[(i-2) h+\frac{h}{2},(i-1) h+\frac{h}{2}\right), \\
0, & \text { otherwise }\end{cases} \\
& \text { for } i=2,3, \ldots, N, j=0,1, \ldots, M \text {, } \\
& \operatorname{HBIBP}_{i, j}(x)=\left\{\begin{array}{ll}
B_{j, M}\left(\frac{2 x}{h}-\frac{2}{h}+1\right), & x \in\left[1-\frac{h}{2}, 1\right), \\
0, & \text { otherwise, }
\end{array} \text { for } i=N+1, j=0,1, \ldots, M .\right.
\end{aligned}
$$

Thus, our new basis is $\left\{\operatorname{HBIBP}_{1,0}, H B I B P_{1,1}, \ldots, H B I B P_{N+1, M}\right\}$. The following section demonstrates the problem of approximating these functions. 


\subsection{Function approximation by HBIBPFs}

In terms of the HBIBP basis a function $u(x)$ can be expressed as follows:

$$
u(x)=\sum_{i=1}^{N+1} \sum_{j=0}^{M} c_{i, j} \cdot H B I B P_{i, j}(x)=C^{T} H B I B P(x),
$$

where

$$
H B I B P(x)=\left[H B I B P_{1,0}, H B I B P_{1,1}, \ldots, H B I B P_{N+1, M}\right]^{T}
$$

and

$$
C=\left[c_{1,0}, c_{1,1}, \ldots, c_{N+1, M}\right]^{T},
$$

we have

$$
C^{T}\langle H B I B P(x), H B I B P(x)\rangle=\langle u(x), H B I B P(x)\rangle
$$

then

$$
C=L^{-1}\langle u(x), H B I B P\rangle,
$$

where $\langle\cdot, \cdot\rangle$ is the standard inner product and $L$ is an $((N+1)(M+1) \times(N+1)(M+1))$ matrix that is said to be the dual matrix; that is,

$$
\begin{aligned}
L & =\langle\operatorname{HBIBP}(x), H B I B P(x)\rangle \\
& =\int_{0}^{1} H B I B P(x) \cdot H B I B P^{T}(x) d x \\
& =\left(\begin{array}{ccccc}
L_{1} & 0 & 0 & \cdots & 0 \\
0 & L_{2} & 0 & \cdots & 0 \\
0 & 0 & L_{3} & \cdots & 0 \\
\vdots & \vdots & \vdots & \ddots & \vdots \\
0 & 0 & 0 & \cdots & L_{n+1}
\end{array}\right),
\end{aligned}
$$

$L_{i}(i=1,2, \ldots, n+1)$ is defined as follows:

$$
\begin{aligned}
\left(L_{1}\right)_{i+1, j+1} & =\int_{0}^{\frac{h}{2}} B_{i, M}\left(\frac{2 x}{h}\right) B_{j, M}\left(\frac{2 x}{h}\right) d x=\frac{h}{2} \int_{0}^{1} B_{i, M}(x) B_{j, M}(x) d x \\
& =\frac{h\left(\begin{array}{c}
M \\
i
\end{array}\right)\left(\begin{array}{c}
M \\
j
\end{array}\right)}{2(2 M+1)\left(\begin{array}{c}
2 M \\
i+j
\end{array}\right)}, \quad \text { for } i, j=0, \ldots, M, \\
\left(L_{r}\right)_{i+1, j+1} & =\int_{(i-2) h+\frac{h}{2}}^{(i-1) h+\frac{h}{2}} B_{i, M}\left(\frac{x}{h}+\frac{3}{2}-i\right) B_{j, M}\left(\frac{x}{h}+\frac{3}{2}-i\right) d x, \quad \text { for } r=2, \ldots, N \\
& =h \int_{0}^{1} B_{i, M}(x) B_{j, M}(x) d x=\frac{h\left(\begin{array}{c}
M \\
i
\end{array}\right)\left(\begin{array}{c}
M \\
j
\end{array}\right)}{(2 M+1)\left(\begin{array}{c}
2 M \\
i+j
\end{array}\right)}, \quad \text { for } i, j=0, \ldots, M,
\end{aligned}
$$




$$
\begin{aligned}
\left(L_{N+1}\right)_{i+1, j+1} & =\int_{1-\frac{h}{2}}^{1} B_{i, M}\left(\frac{2 x}{h}-\frac{2}{h}+1\right) B_{j, M}\left(\frac{2 x}{h}-\frac{2}{h}+1\right) d x \\
& =\frac{h}{2} \int_{0}^{1} B_{i, M}(x) B_{j, M}(x) d x=\frac{h\left(\begin{array}{c}
M \\
i
\end{array}\right)\left(\begin{array}{c}
M \\
j
\end{array}\right)}{2(2 M+1)\left(\begin{array}{c}
2 M \\
i+j
\end{array}\right)}, \quad \text { for } i, j=0, \ldots, M .
\end{aligned}
$$

We can also approximate the function $k(x, t) \in L^{2}([0,1] \times[0,1])$ as follows:

$$
k(x, t)=\operatorname{HBIBP}^{T}(x) \cdot K \cdot H B I B P(t),
$$

where $K$ is an $(M+1)(N+1)$ matrix that we can obtain as follows:

$$
K=L^{-1}\langle H B I B P(x),\langle k(x, t), H B I B P(t)\rangle| L^{-1} .
$$

\section{Solving integral Fredholm equation of second kind via HBIBP function}

In this section, a $H B I B P F$ method is presented to transform the fuzzy Fredholm integral equation of the second kind that is linear (FFIE-2) to two crisp coupled systems. First we consider the fuzzy Fredholm case as the integral Eq. (2.2) in this article with $a=0, b=1$ and $\lambda=1$, where $\tilde{u}(x), \tilde{f}(x) \in L^{2}([0,1))$ and $k(x, t) \in L^{2}([0,1) \times[0,1))$. Our task is to evaluate $H B I B P$ coefficients from the information functions and kernel within the interval $[0,1)$. So, we can add the FFIE-2 parametric form as follows:

$$
\begin{aligned}
& \underline{u}(x, r)=\underline{f}(x, r)+\lambda \int_{a}^{b} \underline{u}(t, r) d t, \\
& \bar{u}(x, r)=\bar{f}(x, r)+\lambda \int_{a}^{b} \bar{U}(t, r) d t .
\end{aligned}
$$

Let us expand $\underline{u}(x, r), \bar{u}(x, r), f(x, r), \bar{f}(x, r)$ and $k(x, t)$ by HBIBPFs (3.1)-(3.7) as follows:

$$
\begin{aligned}
& k(x, t)=H_{B I B P}{ }^{T}(x) \cdot K \cdot H B I B P(t), \\
& \underline{u}(x, r)=\operatorname{HBIBP}^{T}(x) \cdot U 1 \cdot \operatorname{HBIBP}(r), \quad \bar{u}(x, r)=\operatorname{HBIBP}^{T}(x) \cdot U 2 \cdot \operatorname{HBIBP}(r), \\
& f(x, r)=\operatorname{HBIBP}^{T}(x) \cdot F 1 \cdot H B I B P(r), \quad \bar{f}(x, r)=\operatorname{HBIBP}^{T}(x) \cdot F 2 \cdot \operatorname{HBIBP}(r) .
\end{aligned}
$$

Substituting the above equations into Eq. (4.1) yields

$$
\begin{aligned}
& \operatorname{HBIBP}^{T}(x) \cdot U 1 . H B I B P(r) \\
& =\operatorname{HBIBP}^{T}(x) \cdot F 1 \cdot \operatorname{HBIBP}(r) \\
& +\lambda \int_{0}^{1} H B I B P^{T}(x) \cdot K \cdot H B I B P(t) \cdot H B I B P^{T}(t) \cdot U 1 \cdot H B I B P(r) d t, \\
& \operatorname{HBIBP}^{T}(x) \cdot U 1 . H B I B P(r) \\
& =\operatorname{HBIBP}^{T}(x) \cdot F 1 \cdot \operatorname{HBIBP}(r) \\
& +\lambda \cdot \operatorname{HBIBP}^{T}(x) \cdot K\left(\int_{0}^{1} \cdot \operatorname{HBIBP}(t) \cdot \operatorname{HBIBP}^{T}(t) d t\right) U 1 \cdot \operatorname{HBIBP}(r)
\end{aligned}
$$


manipulating Eq. (3.6) and the above equation yields

$$
\begin{aligned}
& \operatorname{HBIBP}^{T}(x) \cdot U 1 \cdot \operatorname{HBIBP}(r) \\
& \quad=\operatorname{HBIBP}^{T}(x) \cdot F 1 \cdot H B I B P(r)+\lambda \cdot H_{B I B P}{ }^{T}(x) \cdot K \cdot D \cdot U 1 \cdot H B I B P(r) .
\end{aligned}
$$

Then

$$
U 1=F 1+\lambda \cdot K \cdot D \cdot U 1 \quad \Rightarrow \quad(I-\lambda \cdot K \cdot D) U 1=F 1 .
$$

Here $I$ is the $(N+1)(M+1)$ identity matrix, then the above equation can be written as follows:

$$
U 1=(I-\lambda \cdot K \cdot D)^{-1} F 1 .
$$

For the solution of the above matrix, we can find the matrix $U 1$. So $\underline{u}(x, r)=H B I B P^{T}(x) \cdot U 1$. $H B I B P(r)$. The same pattern is true of Eq. (4.2).

\section{The convergence characteristics of the proposed method}

This section estimates the error regarding the proposed number method.

Theorem 2 The Fredholm fuzzy integral equation solution using HBIBPFs converges if $M<1$; where $M=\max _{0 \leq x, t \leq 1}|k(x, t)|$.

Proof Assume $\tilde{u}_{E}(x)$ and $\tilde{u}(x)$ are the exact and approximate solutions of Eq. (2.2), respectively. Then

$$
\begin{aligned}
& \begin{aligned}
D\left(\tilde{u}(x), \tilde{u}_{E}(x)\right) & =D\left(\int_{0}^{1} k(x, t) \tilde{u}(t) d t, \int_{0}^{1} k(x, t) \sum_{i=1}^{N+1} \sum_{j=0}^{M} c_{i, j} \cdot H B I B P_{i, j}(x) d t\right) \\
& \leq M \int_{0}^{1} D\left(\tilde{u}(x), \sum_{i=1}^{N+1} \sum_{j=0}^{M} c_{i, j} \cdot H B I B P_{i, j}(x)\right) d t,
\end{aligned} \\
& D\left(\tilde{u}(x), \tilde{u}_{E}(x)\right) \leq M \int_{0}^{1} D\left(\tilde{u}(x), \tilde{u}_{E}(x)\right) d t, \\
& \sup _{0 \leq x \leq 1} D\left(\tilde{u}(x), \tilde{u}_{E}(x)\right) \leq M \sup _{0 \leq x \leq 1} D\left(\tilde{u}(x), \tilde{u}_{E}(x)\right) .
\end{aligned}
$$

Therefore if $M<1$, we will have

$$
\lim _{(n+1)(m+1) \rightarrow \infty}\left(\sup _{0 \leq x \leq 1} D\left(\tilde{u}(x), \tilde{u}_{E}(x)\right)\right)=0 .
$$

\section{Numerical examples}

The accuracy of the proposed solution is demonstrated by solving the following test problems, where all the computations were carried out using the Matlab program (R2018b).

Example 1 Consider the following Fredholm integral linear fuzzy equation [45, 46]:

$$
f(x, r)=-\frac{1}{3} x^{2}+r x^{2}+\frac{1}{3} x+\frac{1}{4} r-\frac{1}{12},
$$


Table 1 The numerical results for Example 1 with $x=0.5, M=2, N=3$

\begin{tabular}{llllll}
\hline$r$ & Exact solution & HBT method [46] & Block-pulse method [45] & Presented method & Absolute error \\
\hline & $\underline{u}(x, r)$ & & & & \\
0 & 0.000000000 & 0.000000000 & 0.007956 & 0.000000000 & $8.58708660 \mathrm{e}-11$ \\
0.1 & 0.050000000 & 0.050000000 & 0.056347 & 0.050000000 & $6.79634155 \mathrm{e}-11$ \\
0.2 & 0.100000000 & 0.100000000 & 0.104737 & 0.100000000 & $5.21343024 \mathrm{e}-11$ \\
0.3 & 0.150000000 & 0.150000000 & 0.153128 & 0.150000000 & $3.83835130 \mathrm{e}-11$ \\
0.4 & 0.200000000 & 0.200000000 & 0.201519 & 0.200000000 & $2.67110889 \mathrm{e}-11$ \\
0.5 & 0.250000000 & 0.250000000 & 0.266040 & 0.250000000 & $1.53477231 \mathrm{e}-12$ \\
0.6 & 0.300000000 & 0.300000000 & 0.314430 & 0.300000000 & $8.80506779 \mathrm{e}-13$ \\
0.7 & 0.350000000 & 0.350000000 & 0.362820 & 0.350000000 & $4.41124914 \mathrm{e}-13$ \\
0.8 & 0.400000000 & 0.400000000 & 0.411210 & 0.400000000 & $2.16571205 \mathrm{e}-13$ \\
0.9 & 0.45000000 & 0.450000000 & 0.359603 & 0.450000000 & $1.03909548 \mathrm{e}-11$ \\
& $\bar{u}(x, r)$ & & & \\
0 & 1.000000000 & 1.000000000 & 1.024160 & 1.000000000 & $1.00612851 \mathrm{e}-11$ \\
0.1 & 0.950000000 & 0.950000000 & 0.975770 & 0.950000000 & $9.72248948 \mathrm{e}-12$ \\
0.2 & 0.900000000 & 0.900000000 & 0.927379 & 0.900000000 & $7.29647454 \mathrm{e}-12$ \\
0.3 & 0.850000000 & 0.850000000 & 0.878988 & 0.850000000 & $2.78301826 \mathrm{e}-12$ \\
0.4 & 0.800000000 & 0.800000000 & 0.830598 & 0.800000000 & $3.81765730 \mathrm{e}-12$ \\
0.5 & 0.750000000 & 0.750000000 & 0.766077 & 0.750000000 & $0.00000000 \mathrm{e}+00$ \\
0.6 & 0.700000000 & 0.700000000 & 0.717986 & 0.700000000 & $3.09223758 \mathrm{e}-12$ \\
0.7 & 0.650000000 & 0.650000000 & 0.669290 & 0.650000000 & $6.18458618 \mathrm{e}-12$ \\
0.8 & 0.600000000 & 0.600000000 & 0.630905 & 0.600000000 & $9.27682375 \mathrm{e}-12$ \\
0.9 & 0.550000000 & 0.550000000 & 0.572514 & 0.550000000 & $4.72755612 \mathrm{e}-11$ \\
\hline & & & &
\end{tabular}

$$
\bar{f}(x, r)=\frac{1}{3} x-x^{2} r-\frac{1}{4} r+\frac{5}{3} x^{2}+\frac{5}{12}
$$

and

$$
k(x, t)=(2 t-1)^{2}(1-2 x), \quad 0 \leq x, t \leq 1 \text { and } \lambda=1 .
$$

The exact solution in this case is given by

$$
\begin{aligned}
& \underline{u}(x, r)=r x, \\
& \bar{u}(x, r)=(2-r) x .
\end{aligned}
$$

Table 1 shows the comparison of the approximate solution for presented method, blockpulse functions [46] for $m=32$, hybrid block-pulse functions and Taylor series (HBT) with $M=4, N=2$ ( $N$ and $M$ are the order of block-pulse functions and Taylor polynomials, respectively) [45] and the exact solution.

Example 2 Consider the following linear Fredholm integral fuzzy equation [47]:

$$
\begin{aligned}
& f_{-}(x, r)=\frac{4}{3} r x-\frac{1}{6} r, \\
& \bar{f}(x, r)=\frac{8}{3} x-\frac{4}{3} r x+\frac{1}{6} r-\frac{1}{3},
\end{aligned}
$$

and

$$
k(x, t)=(2 t-1)^{2}(1-2 x), \quad 0 \leq x, t \leq 1 \text { and } \lambda=1 .
$$


Table 2 The numerical results for Example 2 with $x=0.5, M=2, N=3$

\begin{tabular}{llllll}
\hline$r$ & Exact solution & Presented method & Absolute error & \multicolumn{2}{l}{ Method [47] } \\
\cline { 5 - 6 } & & & HBPBP & $B P$ \\
\hline 0 & $\underline{u}(x, r)$ & & & & \\
0.1 & 0.000000 & 0.000000 & $8.587086597 \mathrm{e}-11$ & 0.001190 & 0.104322 \\
0.2 & 0.050000 & 0.050000 & $6.796341545 \mathrm{e}-11$ & 0.002138 & 0.024766 \\
0.3 & 0.100000 & 0.100000 & $5.213430243 \mathrm{e}-11$ & 0.004753 & 0.025467 \\
0.4 & 0.150000 & 0.150000 & $3.838351303 \mathrm{e}-11$ & 0.005232 & 0.030005 \\
0.5 & 0.200000 & 0.200000 & $2.671108890 \mathrm{e}-11$ & 0.004012 & 0.085691 \\
0.6 & 0.250000 & 0.250000 & $1.534772309 \mathrm{e}-12$ & 0.000891 & 0.070301 \\
0.7 & 0.300000 & 0.300000 & $8.805067786 \mathrm{e}-13$ & 0.002630 & 0.084357 \\
0.8 & 0.350000 & 0.350000 & $4.411249144 \mathrm{e}-13$ & 0.003999 & 0.099502 \\
0.9 & 0.400000 & 0.450000 & $2.165712054 \mathrm{e}-13$ & 0.000248 & 0.054350 \\
1.0 & 0.500000 & 0.450000 & $1.039095476 \mathrm{e}-11$ & 0.003274 & 0.066229 \\
& $\overline{4}(x, r)$ & 0.500000 & $1.773514668 \mathrm{e}-11$ & 0.002265 & 0.012674 \\
0 & 1.000000 & 1.000000 & & & \\
0.1 & 0.950000 & 0.950000 & $1.006128514 \mathrm{e}-11$ & 0.002730 & 0.095432 \\
0.2 & 0.900000 & 0.900000 & $9.722489480 \mathrm{e}-12$ & 0.001276 & 0.083485 \\
0.3 & 0.850000 & 0.850000 & $7.296474536 \mathrm{e}-12$ & 0.001000 & 0.072324 \\
0.4 & 0.800000 & 0.800000 & $2.783018260 \mathrm{e}-12$ & 0.008388 & 0.062431 \\
0.5 & 0.750000 & 0.750000 & $3.817657301 \mathrm{e}-12$ & 0.001192 & 0.059004 \\
0.6 & 0.700000 & 0.700000 & $0.000000000 \mathrm{e}+00$ & 0.002449 & 0.055920 \\
0.7 & 0.650000 & 0.650000 & $3.092237577 \mathrm{e}-12$ & 0.008295 & 0.061354 \\
0.8 & 0.600000 & 0.600000 & $6.184586177 \mathrm{e}-12$ & 0.002286 & 0.075632 \\
0.9 & 0.550000 & 0.550000 & $9.276823754 \mathrm{e}-12$ & 0.000710 & 0.059959 \\
1.0 & 0.500000 & 0.500000 & $4.727556124 \mathrm{e}-11$ & 0.005462 & 0.040971 \\
\hline & & & $5.854872143 \mathrm{e}-11$ & 0.001111 & 0.028821 \\
\hline & & & &
\end{tabular}

The exact solution in this case is given by

$$
\begin{aligned}
& \underline{u}(x, r)=r x, \\
& \bar{u}(x, r)=(2-r) x .
\end{aligned}
$$

Table 2 shows the comparison of the approximate solution for presented method, two methods in [47] (hybrid block-pulse functions and Bernoulli polynomials (HBPBPs) and Bernoulli polynomials (BPs)) with $M=10, N=10$ where $N$ and $M$ are the order of blockpulse functions and Bernoulli polynomials, respectively and the exact solution.

Example 3 Consider the linear fuzzy Fredholm integral equation

$$
\begin{aligned}
& \underline{f}(x, r)=\left(\frac{x}{2}-\frac{1}{3}\right) r, \\
& \bar{f}(x, r)=\left(\frac{x}{2}-\frac{1}{3}\right)(2-r),
\end{aligned}
$$

and

$$
k(x, t)=x+t, \quad 0 \leq x, t \leq 1 \text { and } \lambda=1 .
$$

The exact solution in this case is given by

$$
\begin{aligned}
& \underline{u}(x, r)=r x, \\
& \bar{u}(x, r)=(2-r) x .
\end{aligned}
$$


Table 3 The numerical results for Example 3 with $x=0.5, M=2, N=3$

\begin{tabular}{llll}
\hline$r$ & Exact solution $\underline{\underline{u}}(x, r), \bar{u}(x, r))$ & Presented method & Absolute error \\
\hline 0 & $(0.00000,1.00000)$ & $(0.00000,1.00000)$ & $(1.11378 \mathrm{e}-11,9.60654 \mathrm{e}-12)$ \\
0.1 & $(0.05000,0.95000)$ & $(0.05000,0.95000)$ & $(8.82267 \mathrm{e}-12,7.71254 \mathrm{e}-12)$ \\
0.2 & $(0.10000,0.90000)$ & $(0.10000,0.90000)$ & $(6.79151 \mathrm{e}-12,5.94123 \mathrm{e}-12)$ \\
0.3 & $(0.15000,0.85000)$ & $(0.15000,0.85000)$ & $(5.04428 \mathrm{e}-12,4.29281 \mathrm{e}-12)$ \\
0.4 & $(0.20000,0.80000)$ & $(0.20000,0.80000)$ & $(3.58099 \mathrm{e}-12,2.76719 \mathrm{e}-12)$ \\
0.5 & $(0.25000,0.75000)$ & $(0.25000,0.75000)$ & $(1.53477 \mathrm{e}-12,0.00000 \mathrm{e}+00)$ \\
0.6 & $(0.30000,0.70000)$ & $(0.30000,0.70000)$ & $(8.80507 \mathrm{e}-13,3.09224 \mathrm{e}-12)$ \\
0.7 & $(0.35000,0.65000)$ & $(0.35000,0.65000)$ & $(4.41125 \mathrm{e}-13,6.18459 \mathrm{e}-12)$ \\
0.8 & $(0.40000,0.60000)$ & $(0.40000,0.60000)$ & $(2.16571 \mathrm{e}-13,9.27682 \mathrm{e}-12)$ \\
0.9 & $(0.45000,0.55000)$ & $(0.45000,0.55000)$ & $(1.80808 \mathrm{e}-11,1.17943 \mathrm{e}-10)$ \\
\hline
\end{tabular}

Table 4 The numerical results for Example 4 with $x=0.5, M=2, N=3$

\begin{tabular}{llll}
\hline$r$ & Exact solution $\underline{\underline{u}}(x, r), \bar{u}(x, r))$ & Presented method & Absolute error \\
\hline 0 & $(0.00000,0.50000)$ & $(0.00000,1.00000)$ & $(3.98881 \mathrm{e}-12,4.90985 \mathrm{e}-12)$ \\
0.1 & $(0.02500,0.47500)$ & $(0.05000,0.95000)$ & $(3.11232 \mathrm{e}-12,3.77101 \mathrm{e}-12)$ \\
0.2 & $(0.05000,0.45000)$ & $(0.10000,0.90000)$ & $(2.34518 \mathrm{e}-12,2.77027 \mathrm{e}-12)$ \\
0.3 & $(0.07500,0.42500)$ & $(0.15000,0.85000)$ & $(1.68740 \mathrm{e}-12,1.90765 \mathrm{e}-12)$ \\
0.4 & $(0.10000,0.40000)$ & $(0.20000,0.80000)$ & $(1.13896 \mathrm{e}-12,1.18320 \mathrm{e}-12)$ \\
0.5 & $(0.12500,0.37500)$ & $(0.25000,0.75000)$ & $(7.67386 \mathrm{e}-13,0.00000 \mathrm{e}+00)$ \\
0.6 & $(0.15000,0.35000)$ & $(0.30000,0.70000)$ & $(4.40253 \mathrm{e}-13,1.54612 \mathrm{e}-12)$ \\
0.7 & $(0.17500,0.32500)$ & $(0.35000,0.65000)$ & $(2.20562 \mathrm{e}-13,3.09229 \mathrm{e}-12)$ \\
0.8 & $(0.20000,0.30000)$ & $(0.40000,0.60000)$ & $(1.08286 \mathrm{e}-13,4.63841 \mathrm{e}-12)$ \\
0.9 & $(0.22500,0.27500)$ & $(0.45000,0.55000)$ & $(1.13459 \mathrm{e}-12,1.43827 \mathrm{e}-10)$ \\
\hline
\end{tabular}

Table 3 shows the comparison of the approximate solution for presented method and the exact solution.

Example 4 Consider the linear fuzzy Fredholm integral equation

$$
\begin{aligned}
& \underline{f}(x, r)=\left(\frac{38}{39} x^{2}-\frac{1}{65}+\frac{2}{39}\right) r \\
& \bar{f}(x, r)=\left(\frac{38}{39} x^{2}-\frac{1}{65}+\frac{2}{39}\right)(2-r)
\end{aligned}
$$

and

$$
k(x, t)=\frac{x^{2}+t^{2}-2}{13}, \quad 0 \leq x, t \leq 1 \text { and } \lambda=1
$$

The exact solution in this case is given by

$$
\begin{aligned}
& \underline{u}(x, r)=r x^{2}, \\
& \bar{u}(x, r)=(2-r) x^{2} . \\
& (\underline{u}(x, r), \bar{u}(x, r)) .
\end{aligned}
$$

Table 4 shows the comparison of the approximate solution for presented method and the exact solution. 


\section{Conclusion}

Studying many problems in the applied mathematical many topics requires are required for the solution of the fuzzy integral equations (FIEs). This proposed work introduced a new hybrid method for numerically solving the linear fuzzy integral Fredholm equation. The new hybrid method is based on the combination of Bernstein and improved blockpulse functions. The results obtained from the application of the new method illustrate the superior performance and strong precision of the technique proposed as opposed to other approaches such as HBP, BPFs, HBPBP and BP. Finally, the technique presented in the paper would be of the interest of scientists to solve and study many applied science mathematical problems.

\section{Acknowledgements}

This work was supported by the Natural Science Foundation of China (Grant Nos. 61673169, 11701176, 11626101, 11601485). Praveen Agarwal was very thankful to the SERB (project TAR/2018/000001), DST(project

DST/INT/DAAD/P-21/2019, and INT/RUS/RFBR/308) for their necessary support.

\section{Funding}

This work was supported by the Natural Science Foundation of China (Grant Nos. 61673169, 11701176, 11626101, 11601485).

\section{Availability of data and materials}

All authors declare that no data were used to support this study.

\section{Competing interests}

The authors declare that they have no competing interests.

\section{Authors' contributions}

The authors contributed equally to the writing of this paper. They read and approved the final manuscript.

\section{Author details}

${ }^{1}$ Department of Mathematics, Anand-ICE, Jaipur, India. ${ }^{2}$ Faculty of Science, Menoufia University, Shebin El-Kom, Egypt. ${ }^{3}$ Department of Mathematics, Huzhou University, Huzhou 313000, P.R. China. ${ }^{4}$ Hunan Provincial Key Laboratory of Mathematical Modeling and Analysis in Engineering, Changsha University of Science \& Technology, Changsha 410114, China.

\section{Publisher's Note}

Springer Nature remains neutral with regard to jurisdictional claims in published maps and institutional affiliations.

Received: 1 May 2020 Accepted: 16 September 2020 Published online: 29 September 2020

\section{References}

1. Atanassov, K.T., Rangasamy, P.: Intuitionistic fuzzy sets and interval valued intuitionistic fuzzy sets. Adv. Stud. Contemp. Math. (Kyungshang) 18(1), 1-8 (2009)

2. Nikolova, N., Ivanova, S., Chin, C., Tenekedjiev, K.: Calculation of the Kolmogorov-Smirnov and Kuiper statistics over fuzzy samples. Proc. Jangjeon Math. Soc. 20(2), 269-311 (2017)

3. Shannon, A., Dimitrakiev, D., Sotirova, E., Krawczak, M., Kim, T.: Towards a model of the digital university: generalized net model of appraisal of lecturers with intuitionistic fuzzy estimations. Cybern. Inf. Technol. 9(2), 69-78 (2009)

4. Fidanova, S., Marinov, P., Atanassov, K.: Generalized net models of the process of ant colony optimization with different strategies and intuitionistic fuzzy estimations. Proc. Jangjeon Math. Soc. 13(1), 1-12 (2010)

5. Nanda, S.: On integration of fuzzy mappings. Fuzzy Sets Syst. 32(1), 95-101 (1989)

6. Kaleva, O.: Fuzzy differential equations. Fuzzy Sets Syst. 24, 301-317 (1987)

7. Ralescu, D., Adams, G.: The fuzzy integrals. J. Math. Anal. Appl. 75, 562-570 (1980)

8. Goetschel, R., Voxman, W.: Elementary fuzzy calculus. Fuzzy Sets Syst. 18, 31-43 (1986)

9. Wang, Z:: The autocontinuity of set-function and the fuzzy integral. J. Math. Anal. Appl. 99, 195-218 (1984)

10. Bede, B., Gal, S.G.: Generalizations of the differentiability of fuzzy number-valued function with applications to fuzzy differential equations. Fuzzy Sets Syst. 151, 581-599 (2005)

11. Molabahrami, A., Shidfar, A., Ghyasi, A.: An analytical method for solving linear Fredholm fuzzy integral equations of the second kind. Appl. Comput. Math. 61, 2754-2761 (2011)

12. Maleknejad, K., Shahrezaee, M., Khatami, H.: Numerical solution of integral equations system of the second kind by block-pulse functions. Appl. Math. Comput. 166, 15-24 (2005)

13. Babolian, E., Masouri, Z.: Direct method to solve Volterra integral equation of the first kind using operational matrix with block-pulse functions. Appl. Math. Comput. 220, 51-57 (2008)

14. Maleknejad, K., Sohrabi, S., Berenji, B.: Application of D-BPFs to nonlinear integral equations. Commun. Nonlinear Sci. Numer. Simul. 15, 527-535 (2010) 
15. Maleknejad, K., Mahdiani, K.: Solving nonlinear mixed Volterra-Fredholm integral equations with two dimensional block-pulse functions using direct method. Commun. Nonlinear Sci. Numer. Simul. 16, 3512-3519 (2011)

16. Maleknejad, K., Rahimi, B.: Modification of block pulse functions and their application to solve numerically Volterra integral equation of the first kind. Commun. Nonlinear Sci. Numer. Simul. 16, 2469-2477 (2011)

17. Maleknejad, K., Mordad, M., Rahimi, B.: A numerical method to solve Fredholm-Volterra integral equations I two dimensional spaces using block pulse functions and operational matrix. J. Comput. Appl. Math. (2010)

18. Ordokhani, Y.: Solution of nonlinear Volterra-Fredholm-Hammerstein integral equations via rationalized Haar functions. Appl. Math. Comput. 180, 436-443 (2006)

19. Babolian, E., Maleknejad, K., Roodaki, M., Almasieh, H.: Two dimensional triangular functions and their applications to nonlinear 2D Volterra-Fredholm integral equations. Comput. Math. Appl. 60, 1711-1722 (2010)

20. Maleknejad, K., Almasieh, H., Roodaki, M.: Triangular functions (TF) method for the solution of nonlinear Volterra-Fredholm integral equations. Commun. Nonlinear Sci. Numer. Simul. 15, 3293-3298 (2010)

21. Mirzaee, F., Piroozfar, S.: Numerical solution of the linear two dimensional Fredholm integral equations of the second kind via two dimensional triangular orthogonal functions. J. King Saud Univ., Sci. 22, 185-193 (2010)

22. Kajani, M.T., Vencheh, A.H.: Solving second kind integral equations with hybrid Chebyshev and block-pulse functions. Appl. Math. Comput. 163, 71-77 (2005)

23. Wang, X.T., Li, Y.M.: Numerical solutions of integro-differential systems by hybrid of general block-pulse functions and the second Chebyshev polynomials. Appl. Math. Comput. 209, 266-272 (2009)

24. Maleknejad, K., Kajani, M.T.: Solving second kind integral equations by Galerkin methods with hybrid Legendre and block-pulse functions. Appl. Math. Comput. 145, 623-629 (2003)

25. Hashemzadeh, E., Maleknejad, K., Basirat, B.: Hybrid functions approach for the nonlinear Volterra-Fredholm integral equations. Proc. Comput. Sci. 3, 1189-1194 (2011)

26. Marzban, H.R., Tabrizidooz, H.R., Razzaghi, M.: A composite collection method for the nonlinear mixed Volterra-Fredholm-Hammerstein integral equation. Commun. Nonlinear Sci. Numer. Simul. 16, 1186-1194 (2011)

27. Maleknejad, K., Mahmoudi, Y.: Numerical solution of linear Fredholm integral equation by using hybrid Taylor and block-pulse functions. Appl. Math. Comput. 149, 799-806 (2004)

28. Asady, B., Kajani, M.T., Vencheh, A.H., Heydari, A.: Solving second kind integral equations with hybrid Fourier and block-pulse functions. Appl. Math. Comput. 160, 517-522 (2005)

29. Maleknejad, K., Mohsenyzadeh, M., Hashemizadeh, E.: Hybrid orthonormal Bernstein and block-pulse functions for solving Fredholm integral equations. In: Proceedings of the World Congress on Engineering, vol. 1, pp. 91-94 (2013)

30. Masouri, Z.: Numerical expansion-iterative method for solving second kind Volterra and Fredholm integral equations using block-pulse functions. Adv. Comput. Tech. Electromagn. 20, 7-17 (2012)

31. Alipour, M., Baleanu, D., Babaei, F.: Hybrid Bernstein block-pulse functions method for second kind integral equations with convergence analysis. Abstr. Appl. Anal. 2014, Article ID 623763 (2014)

32. Rao, G.P.: Piecewise Constant Orthogonal Functions and Their Application to System and Control. Springer, Berlin (1983)

33. Mohan, B.M., Datta, K.B.: Orthogonal Function in Systems and Control (1995)

34. Chen, C.F., Tsay, Y.T., Wu, T.T.: Walsh operational matrices or fractional calculus and their application to distributed systems. J. Franklin Inst. 303, 267-284 (1977)

35. Sannuti, P.: Analysis and synthesis of dynamic systems via block pulse functions. Proc. IEEE 124, 569-571 (1977)

36. Maleknejad, K., Hashemzadeh, E., Ezzati, R.: A new approach to the numerical solution of Volterra integral equations by using Bernstein approximation. Commun. Nonlinear Sci. Numer. Simul. 16, 647-655 (2011)

37. Doha, E.H., Bhrawy, A.H., Saker, M.A.: Integrals of Bernstein polynomials: an application for the solution of high even-order differential equations. Appl. Math. Lett. 24, 559-565 (2011)

38. Mandal, B.N., Bhattacharya, S.: Numerical solution of some classes of integral equations using Bernstein polynomials Appl. Math. Comput. 190, 1707-1716 (2007)

39. Maleknejad, K., Hashemizadeh, E., Basirat, B.: Computational method based on Bernestein operational matrices for nonlinear Volterra-Fredholm-Hammerstein integral equations. Commun. Nonlinear Sci. Numer. Simul. 17(1), 52-61 (2012)

40. Maleknejad, K., Basirat, B., Hashemizadeh, E.: A Bernstein operational matrix approach for solving a system of high order linear Volterra-Fredholm integro-differential equations. Math. Comput. Model. 55, 1363-1372 (2012)

41. Mirzaee, F.: Numerical solution of system of linear integral equations via improvement of block-pulse functions. J. Math. Model. 4(2), 133-159 (2016)

42. Maleknejad, K., Khodabin, M., Hosseini Shekarabi, F.: Modified block pulse functions for numerical solution of stochastic Volterra integral equations. J. Appl. Math. 2014, Article ID 469308 (2014)

43. Ramadan, M.A., Osheba, H.S.: A new hybrid orthonormal Bernstein and improved block-pulse functions method for solving mathematical physics and engineering problems. Alex. Eng. J. (2020). In press. https://doi.org/10.1016/j.aej.2020.06.014

44. Mirzaee, F., Paripour, M., Komak Yari, M.: Numerical solution of Fredholm fuzzy integral equations of the second kind via direct method using triangular functions. J. Hyperstruct. 1(2), 46-60 (2012)

45. Ghanbari, M., Toushmalni, R., Kamrani, E.: Numerical solution of linear Fredholm fuzzy integral equation of the second kind by block-pulse functions. Aust. J. Basic Appl. Sci. 3(3), 2637-2642 (2009)

46. Baghmisheh, M., Ezzati, R.: Numerical solution of nonlinear fuzzy Fredholm integral equations of the second kind using hybrid of block-pulse functions and Taylor series. Adv. Differ. Equ. 2015, 51 (2015)

47. Ezzati, R., Sadatrasoul, S.M.: Two hybrid and non-hybrid methods for solving fuzzy integral equations based on Bernoulli polynomials. J. Fuzzy Set Valued Anal. 2016, 31-42 (2016) 